\title{
Retos para las \\ intervenciones psicológicas y psicosociales en Colombia en el marco de la implementación de los acuerdos de paz entre el gobierno y las FARC-EP1
}

\author{
Miguel Gutiérrez-Peláez² \\ Artículo de la revista Avances en psicología latinoamericana \\ de la Universidad del Rosario.
}

\footnotetext{
${ }^{1}$ Fuente original en Gutiérrez-Peláez, M. (2017). Retos para las intervenciones psicológicas y psicosociales en Colombia en el marco de la implementación de los acuerdos de paz entre el gobierno y las FARC-EP. Avances en Psicología Latinoamericana, 35(1), 1-8. Recuperado de www.redalyc.org/pdf/799/79949625001.pdf. Agradecimientos especiales a Miguel Gutiérrez-Peláez, así como la editorial de la Universidad del Rosario, por permitirnos publicar este artículo tan relevante para la temática del número. Todas notas pie de páginas fueron agregadas por el Equipo Editorial de la revista Trans-pasando Fronteras.

2 Master en psicoanálisis (Buenos Aires 2007) y Doctor en Psicología (Buenos Aires 2010), vinculado a la Universidad del Rosario.

${ }^{3}$ Psicóloga con Magister en Educación.
} 
Desde el inicio de los diálogos de paz de la Habana en el 2012, que son sin lugar a dudas uno de los hitos más importantes de la historia reciente de nuestro país, la psicología se ha posicionado como un interlocutor necesario, activo y propositivo, tanto desde agremiaciones, como el Colegio Colombiano de Psicólogos (Colpsic) y la Asociación Colombiana de Facultades de Psicología (Ascofapsi), como desde las universidades y desde la labor de diversos organismos y las acciones, a veces individuales, de un gran número de psicólogos que laboran e intervienen desde organismos estatales y privados que inciden directamente tanto en la atención y en la elaboración de políticas públicas, protocolos, estrategias de intervención, consultorías, etc. Lo que se ha movilizado socialmente a partir del Ilamado proceso de paz ha hecho patente, sin embargo, que a pesar del gran número de psicólogos egresados (cerca de 100 000; Colpsic, noviembre, 2016) y en formación que hay en el país, la mayoría carece de las competencias y habilidades para responder a los encargos y demandas sociales actuales.

Esto hace patente tanto la gran urgencia y responsabilidad social de la psicología, como la altísima responsabilidad de los programas de psicología, que son los que tienen la tarea de formar a los profesionales de este campo. Se ha hecho evidente, también, que el saber y hacer de la psicología no es un terreno que competa solamente a los psicólogos, sino que tiene una influencia en el entendimiento y campo de acción de otras disciplinas que también intervienen en campos derivados del conflicto armado y su violencia.

Aparte de las consecuencias psicopatológicas y de salud mental derivadas del conflicto armado, que han sido consignadas en diferentes estudios (Ministerio de la Protección Social, 2015), 
hay otra serie de exigencias o retos psicológicos a los que estarán enfrentados los colombianos en el 2017 y en los años posteriores, ante los cuales vale la pena detenerse y tratar de extraer lo que la disciplina y profesión psicológica pueden aportar. De las muchísimas que sería posible ubicar, quisiera enunciar las siguientes para, después, ofrecer unas reflexiones que permitan orientar los modos posibles de intervenir frente a ello:

- El reto psicológico de reconocer que hay pérdidas irreparables, que van desde la aceptación de los contenidos de los acuerdos de paz entre el gobierno y las FARC, hasta las consecuencias más crudas de la violencia vivida durante estos más de cincuenta años, y los corolarios de ello para las víctimas.

- El reto psicológico de soportar, y lidiar con, la intensificación de una serie de conflictos políticos y sociales que han estado opacados por la lucha armada. Es muy probable que, lejos de reducirse los conflictos sociales durante el 2017, estos aumenten al ser corrido el manto de conflicto armado que los encubría.

- El reto psicológico de enfrentarse, cara a cara, con la complejidad del comportamiento humano y con la evidencia de que el conflicto armado no ha correspondido a un conflicto entre buenos y malos (Briole, 2015). ¿Cómo conciliar la bondad, los ideales y el carisma de un sujeto con el hecho de haber cometido delitos atroces?

- El reto psicológico de mantener vivo el despertar de la sociedad civil frente al conflicto armado y el proceso de paz, pasando de la pasividad (en la aceptación o no de las propuestas de otros) a la actividad (proponiendo soluciones y ampliando el marco de posibilidades), de la recepción de propuestas, a ser agentes que proponen soluciones (Gutiérrez-Peláez \& Herrera-Pardo 2016b). 
Los estudiantes han tenido un papel protagónico en la etapa final de la firma de los acuerdos de paz y mantener ese activismo será su reto en los años por venir. Por otro lado, la sociedad civil se enfrenta a la exigencia psicológica de reconocer que su pasividad durante las décadas de conflicto armado no los ha hecho neutrales, sino más bien permisivos de los acontecimientos atroces de la guerra.

- El reto psicológico de reconocer el lugar del otro, de la alteridad, dándole voz y asumiendo la existencia de un disenso sin armas.

- El reto psicológico de entender que la llamada "paz" o "posconflicto" no es la ausencia de conflicto, sino una elevación del propio conflicto, sumado al reto, además, de poder enfrentarlo con la palabra y por las vías democráticas.

¿Cómo hacer frente a estos retos psicológicos? ¿Cómo orientarnos frente a las intervenciones psicológicas y psicosociales que pueden implementarse en diferentes contextos en este momento histórico? ¿Cómo mantener abierta una conversación en diferentes sectores sociales y en la comunidad que permita el discurrir de la complejidad de los contenidos que están en juego?

\section{Una nueva conversación}

El arte de conversar implica, necesariamente, la atribución de un saber en el otro. Esa atribución de saber en el otro requiere, a su vez, de una posición de no saber de parte de uno. Si yo tengo todas las verdades de mi lado, no hay lugar para alojar la palabra del otro, a menos que esa palabra replique las mías. Para poder conversar, debo despojarme de mi verdad y asumir que el otro puede enseñarme algo a mí. 
Es la invitación que podemos hacerles a los diferentes profesionales que realizan intervenciones psicosociales y es lo que hemos aprendido de nuestro trabajo clínico y de campo y de las intervenciones a profundidad realizadas en el Centro de Estudios Psicosociales (Cepso) de la Escuela de Medicina y Ciencias de la Salud (EMCS) de la Universidad del Rosario: justamente que es posible dejarnos enseñar del otro, de todo otro, y allí incluimos al político, al soldado, al guerrillero, al desmovilizado, al civil, al niño, al paramilitar. Esto lo hemos aprendido de nuestro propio trabajo clínico, dejándonos enseñar tanto de personas sanas como de personas con diagnósticos de enfermedades mentales graves y sus formas de sufrimiento.

No es una poción de falsa humildad para llevar a que se produzca la palabra del otro o su confianza; es una posición ética profunda y legítima que encontramos como necesaria: el poder extraer de la propia persona un saber sobre lo que lo atormenta, sobre sus dolores y sus virtudes. Nos ha interesado conocer el propio saber que tienen las personas y las comunidades, y a partir de allí acompañarlas en el proceso de construir sus soluciones de aliviar sus padecimientos y alcanzar sus metas, legitimando y reconociendo aquellas acciones y decisiones que han tomado y que, muchas veces, han sido muy exitosas, pero que en algunos casos requieren la ayuda de un otro para responder de una mejor manera a una nueva situación, con un costo emocional menor para el sujeto o la comunidad.

Las intervenciones psicológicas y psicosociales que se implementen en diferentes comunidades y regiones de nuestro país tienen que tener en cuenta las soluciones que cada una de esas comunidades ha construido para responder a sus problemáticas. No hay un metadiscurso o un plan de intervención que pueda pre- 
scindir del saber de cada comunidad y de cada sujeto (GutiérrezPeláez \& Herrera-Pardo, 2016a). Es posible que nuestra posición ética y nuestra escucha entrenada, más que nuestro saber, puedan aportar a esa construcción de intervenciones exitosas. La conversación con el otro requiere mantener ese lugar vacío para que discurra y circule la palabra y la verdad del otro.

\section{Producción y reconocimiento de la rareza del otro}

Es necesario, entonces, para la planeación e implementación de cualquier intervención psicosocial en este tránsito de un conflicto con armas a uno sin armas reconocer las estrategias que han sido exitosas en las diferentes regiones y, a partir de allí, crear las intervenciones psicológicas y psicosociales posibles. Son útiles, sin duda, los modelos y miradas que podemos traer de otros países. Eso permite iluminar nuestra realidad y aprender de las experiencias de los otros. Pero seguramente la situación de Colombia tiene sus coordenadas singulares y requiere por ello de la producción e invención de soluciones e intervenciones igualmente singulares. La experiencia nos ha mostrado que esto es posible solamente teniendo en cuenta las iniciativas que han surgido de las comunidades y los sujetos.

En coyunturas tan dramáticas como la de nuestro país, no es en una conversación de unos pocos que puede desplegarse la complejidad de su historia. Más que un diálogo, es una polifonía de voces que revela que la verdad no es un saber homogéneo y que es a varias manos que hay que escribir esa historia. Los griegos hablaban de un palimpsesto, que es un manuscrito que conserva en su superficie grabadas las huellas de trazos anteriores, borrados para introducir una nueva escritura. En el siglo XIX, y con la ayuda 
de nuevas técnicas, fueron recuperadas las escrituras antiguas de los papiros. Así, el palimpsesto ha ido recobrando, en una misma superficie, una multiplicidad de escrituras distintas y, diríamos, es esa la verdad ampliada intrínseca al mismo papiro. Del mismo modo, la historia de Colombia es un palimpsesto, y tendremos que acostumbrarnos y encontrar la virtud en la imposible unificación de los trazos que en él se graban.

Es innegable la virtud del dispositivo jurídico para la construcción de determinados hechos y para edificar una versión de la historia. Pero no es posible escribir una verdad que tenga la pretensión de hablar por todos aquellos que han atravesado una historia particular, aun los mismos hechos, colindantes en tiempo y espacio. Hay una necesidad -y es de gran importancia- de que existan testimonios que aporten a una reconstrucción jurídica de la verdad. Pero se requieren también otros dispositivos que permitan escuchar las palabras emitidas por un cuerpo visceral, emocional y pasional. Esos testimonios que hemos estudiado a profundidad, en los que la falta de coherencia racional de los hechos, las imprecisiones temporales, la ambivalencia afectiva no suponen una serie de errores a corregir, sino la verdad misma de la vivencia emocional de los hechos, el modo singular como unos hechos determinados, dolorosos, traumáticos, violentos, han marcado la historia de un cuerpo (Gutiérrez-Peláez, 2008a, 2009, 2015a).

\section{Las invenciones singulares}

La incertidumbre a la que nos enfrentamos actualmente en el proceso de implementación de los acuerdos no debe hacernos perder de vista lo que hemos ganado como país a lo largo de este proceso. La apuesta exigente de este momento histórico es que, 
para implementar cualquier acuerdo en el corazón de la sociedad colombiana, hay que irnos paulatinamente abriendo a la polifonía de voces que trae para cada uno el riesgo de abandonar la consistencia sólida a la que aferramos una idea de la verdad. Es la nueva ética que implica desanudar las categorías de buenos y malos que armaban la lógica de los discursos a través de los cuales interpretamos la realidad.

Con frecuencia, el profesional de la salud mental está entrenado para encontrar lo que en un sujeto se suma a una nosología determinada. Debemos estar advertidos de lo que hemos denominado en el Centro de Estudios Psicosociales (CEPSO) "la falsa unificación de las muestras" de determinados modos de extraer evidencias empíricas, por ejemplo, tomar como poblaciones homogéneas a las FARC, a las víctimas, a los reinsertados, a los miembros de las fuerzas armadas, etc. Por el contrario, la evidencia nos ha llevado a encontrar en los sujetos y en las comunidades aquello que de ellos está por fuera de toda nosología posible. ¿Cómo entrenarnos para ver lo diferente del otro y no su mismidad (Gutiérrez-Peláez, 2008b)? ¿No aquello que lo suma a un concepto o categoría superior, sino aquello que el poeta René Char llamaba su "legítima rareza"?

Confieso mi sesgo y mi fascinación personal por las invenciones singulares y por los fenómenos que resaltan un funcionamiento novedoso e inédito, como aquel pingüino del documental de Werner Herzog (2008) que se escapa de la manada y sale indetenible en una dirección contraria, para encontrar la muerte en las montañas. Eso que no entendemos solemos Ilamarlo locura y las categorías de normal y anormal, así como las de buenos y malos, son modos habituales con los que enmarcamos los fenómenos humanos y a través de los cuales propagamos modos de segregación. 
Es, sin duda, una pregunta de una gran pertinencia: ¿cómo se hace ciencia de lo singular?

Si bien es fascinante, como lo revelan muchos estudios contemporáneos, el modo como determinadas situaciones producen efectos comunes en una población o grupo de personas, también es fascinante cómo, a pesar de esas situaciones, rasgos, predisposiciones y causas comunes, un sujeto, un organismo o un cuerpo producen un efecto distinto. Es fascinante, por ejemplo, la implicación de los hemisferios cerebrales temporales en toda la maquinaria del lenguaje. Y también es fascinante, como me lo enseñó hace muchos años un niño de 7 años, que es posible tener un aparato del lenguaje, motor y semántico, intacto con una alteración en el neurodesarrollo de los lóbulos temporales como secuela de una hipoxia al nacer.

Es fascinante descubrir creaciones singulares en sujetos aun en las circunstancias más adversas. Me lo enseñó también un niño, hace más de 15 años, en una de mis primeras experiencias como psicoterapeuta. Este niño, al que llamaré Juan para efectos de este escrito, de 11 años, estaba internado en la Unidad de Cuidados Intensivos por cuarta recaída de Guillan Barré. Había perdido casi todas sus funciones motoras, excepto el movimiento de los ojos, y debía estar 24 horas al día inmóvil y conectado a un respirador. Tuve la oportunidad de enseñarle a jugar un poco de ajedrez y también parqués, y en este último resultó ser un contrincante excepcional. Juan había aprendido que, con un gran esfuerzo, podía inhibir todavía algunos de sus músculos torácicos. Así, cuando lograba vencerme, se esforzaba por producir una contracción muscular, lo que disparaba las alarmas del respirador y también la alarma de las enfermeras. Pero no era para alarmarse, sino más bien para conmoverse: esa alarma de la máquina era el 
grito de triunfo de Juan, su celebración y su aullido para el mundo. Imposibilitado de alzar su voz y de ser escuchado, había logrado extraer el pitido de la máquina y, a través de él, colar su emoción. Era su invención motora; no tenía ni un tercio de caballos de fuerza, pero era más poderosa que una locomotora. Juan había encontrado en mi mirada un cómplice para su travesura y era un verdadero acontecimiento ver cómo sonreía con sus ojos ante su picardía y ante el regaño de la enfermera. Juan nos muestra cómo, frente a las condiciones más adversas, es posible que haya espacio para invenciones singulares, inéditas, que dignifiquen al sujeto y viralicen esa dignificación a otros espacios.

\section{Los peligros de la segregación}

Es un gran paso histórico el que está dando Colombia. Pero lo negociado en la Habana y aprobado en los dispositivos democráticos gubernamentales no resuelve nada por sí mismo. Para que sea implementable cualquier acuerdo, se requiere un nuevo diálogo, una nueva conversación entre los colombianos. El proceso de paz se esfuerza por sentar las bases para un mejor país y apunta a que sea mejor la Colombia de las generaciones venideras que la nuestra. Pero solo los acuerdos que se alcancen no salvan a nuestros hijos de un mundo peor. Se requiere una decisiva transformación de nuestros modos de relación.

¿Podremos animarnos a un verdadero aprendizaje de las diferencias? Esa, considero, ha sido la posición al interior del Centro de Estudios Psicosociales (Cepso). El saber no lo tienen los profesores ni los estudiantes ni los libros. El saber está por producirse en la conversación entre sujetos que han dejado en su seno un no saber y han hecho de ese agujero estructural la fuente para la 
producción de un nuevo saber, cada uno desde su particularidad, desde su formación profesional, su historia de vida, sus convicciones, sus goces singulares, en últimas, desde su legítima rareza.

En situaciones como las violencias a las que nos enfrentamos hoy en día en el mundo, hay que preguntarse si corresponden a nuevos fenómenos o si son más bien nuevas versiones de las locuras humanas de siempre. Muchas veces la tecnología y los desarrollos dramáticos que vemos y que transforman nuestras relaciones sociales nos hacen creer que el mundo se creó ayer. Pero, como lo sugería recientemente Indart (2016), hay un funcionamiento segregativo antiguo, desde que hay las pretensiones de fundar órdenes sociales nuevos, a través del cual designamos un "nosotros" que deja automáticamente por fuera unos "otros". Para poder decir "todos nosotros", para producir ese conjunto, necesitamos excluir algo, dejar algunos por fuera. Así, cada vez que hay un "nosotros", hay personas que quedan desenlazadas de ese vínculo social. Ante el vértigo que produce la disolución de ese vínculo social, los individuos pueden lanzarse a invocar ideales que les ofrezcan una nueva forma de lazo con esos otros excluidos, o a elegir al candidato de turno que encarna ese ideal. Y esa nueva forma de lazo, que es un nuevo "nosotros", deja automáticamente unos otros segregados. El conjunto de los que votaron por el "sí", por ejemplo, es un "nosotros" que excluye los que votaron por el "no", y viceversa. Es la lógica segregativa, incrustada en el lenguaje mismo, y que adquiere su dimensión más voraz cuando hay amenazas reales de disolución del vínculo social. Y hay también sujetos que permanecen en la periferia del lazo social, lo que produce, eso sí, respuestas inéditas de la época y fenómenos humanos que nos resultan incomprensibles, algunos de una violencia abrumadora (ver, por ejemplo, los modos contemporáneos del terrorismo: Gómez, López-Rodríguez, Vázquez, Paredes, \& 
Martínez, 2016; Post, McGinnis, \& Moody, 2014; Stafford, 2015; o el fenómeno hikikomori: Gutiérrez-Peláez, 2015b; Koyama et al., 2010; Laurent, 2014; Rosenthal \& Zimmerman, 2013; Suwa \& Susuki, 2013; Zielenziger, 2006, para nombrar unos que han ido aumentando su incidencia en el mundo).

¿Cómo escapar a esa vicisitud que se encuentra en germen en el lenguaje mismo (Heller-Roazen, 2008)? ¿Cómo evocar un conjunto que no sea cerrado, que sea no-todo, que deje un lugar abierto para que pueda enlazarse también, desde su rareza, aquel que no aloja los atributos del conjunto? ¿Cómo mantener cierta polisemia del lenguaje y que, cuando hablemos de "los colombianos", eso no sea una definición cerrada de un "nosotros", sino que en ella contenga toda la diversidad, no solo de aquello que nos hace comunes, sino de aquello que nos hace radicalmente diferentes? ¿Cómo dejar la puerta abierta?

La profesora Bruna Zani (2016), exdecana de la Facultad de Psicología de la Universidad de Bolonia, hablaba recientemente de la existencia de lo que denominaba "metas superordinadas" (Pisor \& Gurven, 2016; Prati, Mazzoni, Cicoqnani, Albanesi, \& Zani, 2016; Stephen, 2016). La doctora Zani se preguntaba si era posible que la guerrilla de las FARC y el Estado, enfrentados durante tantas décadas, pudieran tener como meta superordinada una meta común. Incluso, que esa meta superordinada acogiera y agrupara otros grupos enfrentados de nuestro país. ¿Podría ser una meta superordinada de los colombianos la producción de una sociedad en la que coexista la alteridad radical? Lo difícil de esa meta no debería inhibirnos a ponernos al servicio de hacerla posible, de volcarnos y tender a ella. 


\section{Indicaciones para las intervenciones psicológicas y psicosociales}

Según lo comentado, las intervenciones psicológicas y psicosociales deben necesariamente reconocer y construirse a partir de las invenciones exitosas de cada sujeto y de cada comunidad. Las intervenciones tienen que responder y valorar esa legítima rareza que ha sido la respuesta singular que han dado a los fenómenos y vivencias de su vida. Será partiendo de ese trabajo que podremos ver si en algo nuestro saber y nuestro entrenamiento pueden aportar para hacer un poco más exitosas esas intervenciones que han surgido de los sujetos o de la comunidad.

La escucha que ofrezcamos también a los sujetos y a la comunidad no tiene por quétener la pretensión de reconstruir una verdad de los hechos históricos, jurídicos o periodísticos. Nos interesa la verdad del sujeto y podemos permitir que toda la complejidad de su vivencia psíquica discurra en los dispositivos psicosociales que les ofrezcamos.

Por último, debemos también prescindir de categorías que establezcan un "nosotros" que deje por fuera una porción de la población, o, por lo menos, estar muy advertidos de los efectos segregativos que pueden tener nuestras palabras y nuestras intervenciones. Así, animados al encuentro con lo legítimamente raro del otro, podremos, en esta nueva emocionalidad y con nuestro estado mental transicional (Fergusson, junio, 2016), abrirnos a un nuevo encuentro con el otro y, en esa conversación, darle una voz hasta ahora silenciada. 


\section{Referencias}

Briole, G. (2015). En las fauces de la guerra: arrancamiento. En M. H. Brousse (Ed.). El psicoanálisis a la hora de la guerra (pp. 101106). Buenos Aires: Tres Haches.

Colegio Colombiano de Psicólogos (Colpsic) (noviembre, 2016). Analizando un país en paz. El Tiempo. Recuperado de http://www.eltiempo.com/contenido-comercial/publirreportaje/ analizando-un-pais-en-paz-/16760711

Fergusson, A. (2016). Las consecuencias psicológicas de los acuerdos de la Habana. Hacia una nueva fase del conflicto colombiano en medio de un estado mental transicional. II Simposio de las emociones en escenarios de conflicto: Una mirada interdisciplinaria al posacuerdo. Bogotá: Universidad del Rosario.

Gómez, A., López-Rodríguez, L., Vázquez, A., Paredes, B., \& Martínez, M. (2016). Morir y matar por un grupo o unos valores. Estrategias para evitar, reducir o erradicar el comportamiento grupal extremista. Anuario de Psicología Jurídica, 26, 122-129.

Gutiérrez-Peláez, M. (2008a). La noción ferencziana de "Orpha”. Psicoanálisis, 2(3), 285-290.

Gutiérrez-Peláez, M. (2008b). ¿Qué un otro otro? Ideas y Valores. Revista Colombiana de Filosofía, 136, 105-115.

Gutiérrez-Peláez, M. (2009). Trauma theory in the 1931 and 1932 writings of Sandor Ferenczi. International Journal of Psychoanalysis, 90, 1217-1233. 
Gutiérrez-Peláez, M. (2015a). Ferenczi's anticipation of the traumatic dimension of language: A meeting with Lacan. Contemporary Psychoanalysis, 51(1), 137-154. doi: 10.1080/00107530.2015.957255

Gutiérrez-Peláez, M. (2015b). Hikikomori. Consecuencias, 16. Recuperado de http://www.revconsecuencias.com.ar/ediciones/016/template.php?file=arts/Variaciones/Hikikomori.html

Gutiérrez-Peláez, M. \& Herrera-Pardo, E. (2016a). Ambiente, trauma e innovaciones técnicas: tres articulaciones entre Donald W. Winnicott y Sándor Ferenczi. Revista Colombiana de Psiquiatría (en prensa). doi: http://dx.doi.org/10.1016/j.rcp.2015.12.001

Gutiérrez-Peláez, M. \& Herrera-Pardo, E. (2016b). La escucha del Otro como aporte a la construcción de paz. En N. Molina-Valencia \& W. López-López (Eds.). Trabajando en la paz y en la reconciliación (pp. 58-59). Bogotá, Colombia: Colegio Colombiano de Psicólogos.

Heller-Roazen, D. (2008). Echolalias. On the forgetting of language. Nueva York: Zone Books.

Herzog, W. (2008). Encounters at the end of the world. Estados Unidos: Discovery Films.

Idart, J. C. (2016). Sobre la política del miedo y el discurso de Donald Trump. En Mediodicho (pp. 84-86). Córdoba: EOL.

Koyama, A., Miyake, Y., Kawakami, N., Tsuchiya, M., Tachimori, H., \& Takeshima, T. (2010). Lifetime prevalence, psychiatric comorbidity and demographic correlates of "hikikomori" in a community population in Japan. Psychiatry Res, 176, 69-74. 
Laurent, E. (2014). El sujeto de la ciencia y la distinción femenina. Letras lacanianas, 8, 12-24.

Ministerio de la Protección Social (2015). Encuesta nacional de salud mental 2015. Tomo 1, Colombia. Recuperado de http:// www.odc.gov.co/Portals/1/publicaciones/pdf/consumo/estudios/nacionales/CO031102015-salud_mental_tomol.pdf

Pisor, A. C. \& Gurven, M. (2016). Corruption and the other(s): Scope of superordinate identity matters for corruption permissibility. PlosONE, 10(12), e0144542. doi: 10.1371/journal. pone.0144542

Prati, G., Mazzoni, D., Cicoqnani, E., Albanesi, C., \& Zani, B. (2016). Evaluating the persuasiveness of an HIV mass communication campaign using gain-framed messages and aimed at creating a superordinate identity. Health Communication, 9, 1 097-1 104. doi: 10.1080/10410236.2015.1040983

Post, J. M., McGinnis, C., \& Moody, K. (2014). The changing face of terrorism in the 21st century: The communications revolution and the virtual community of hatred. Behavioral Sciences and the Law, 32, 306-334.

Rosenthal, B. \& Zimmerman, L. (2013). Hikikomori. The Japanese phenomenon, policy, and culture. International Journal of Mental Health, 41(4), 82-95.

Stafford, M. (2015). Is there a psychoanalytic response to terrorism? Or "To win the pure prestige of horror". Issues In Psychoanalytic Psychology, 37, 17-24. 
Stephen, R. (2016). Superordinate and subgroup identities as predictors of peace and conflict: The unique content of global citizenship identity. Peace and Conflict: Journal of Peace Psychology. doi: 10.1037/pac0000208

Suwa, M. \& Susuki, K. (2013). The phenomenon of "hikikomori" (social withdrawal) and the socio-cultural situation in Japan today. Journal of Psychopathology, 19, 191-198.

Zani, B. (2016). Sentido de comunidad entre cohesión social y conflicto. Simposio «Psicología, Paz y Conflicto». Bogotá: Universidad del Rosario.

Zielenziger, M. (2006). Shutting out the sun: How Japan created its own lost generation. Nueva York: Talese. 